\title{
Two-Photon Decays of Charmonia from Lattice QCD
}

\author{
Jozef J. Dudek* and Robert G. Edwards \\ Jefferson Laboratory MS 12H2, 12000 Jefferson Avenue, Newport News, Virginia 23606, USA
}

(Received 12 July 2006; published 23 October 2006)

\begin{abstract}
We make the first calculation in lattice QCD of two-photon decays of mesons. Working in the charmonium sector, using the Lehmann-Symanzik-Zimmermann reduction to relate a photon to a sum of hadronic vector eigenstates, we compute form factors in both the spacelike and timelike domains for the transitions $\eta_{c} \rightarrow \gamma^{*} \gamma^{*}$ and $\chi_{c 0} \rightarrow \gamma^{*} \gamma^{*}$. At the on-shell point, we find approximate agreement with experimental world-average values.
\end{abstract}

DOI: 10.1103/PhysRevLett.97.172001

The two-photon widths of charmonia have attracted the attention of a range of a theoretical and experimental techniques. Within perturbative QCD, the two-photon branching fraction is believed to give access to the strong coupling constant at the charmonium scale though cancellation of nonperturbative factors, while in quark models and the effective field theory nonrelativistic quantum chromodynamics (NRQCD), two-photon widths have been proposed as a sensitive test of the corrections to the nonrelativistic approximation. Experimental measurements are diverse, coming from, on the one hand, two-photon fusion at $e^{+} e^{-}$machines with subsequent reconstruction of the charmonium in light hadrons and, on the other, $p \bar{p}$ annihilation to charmonium with decay to real $\gamma \gamma$ pairs being detected. Improvements in extracted branching fractions from both methods will soon arrive via more accurate measurements from the Belle, BABAR, and CLEO-c Collaborations of exclusive hadronic $\eta_{c}$ decays.

Despite the considerable theoretical and experimental interest, there has not yet been an $a b$ initio estimation of two-photon widths in charmonia. In this Letter, we address this issue using a novel application of lattice QCD.

Conventionally, lattice QCD calculations involve the evaluation of the matrix element of an operator between
PACS numbers: $12.38 . \mathrm{Gc}, 12.40 . \mathrm{Vv}, 13.25 . \mathrm{Gv}$

hadron states. The hadrons are induced using interpolating fields (some combination of quark and gluon fields) with the appropriate hadron quantum numbers. However, these fields will typically have overlap with many excited states which have the same external quantum numbers so that, in order to isolate the ground states, the interpolating fields are taken out to Euclidean times far from the operator (and each other). Such an approach will clearly not work if the initial or final state contains no hadron, as is the case for two-photon decays since the photon is not an eigenstate of QCD. In this case, we can adopt a slightly more sophisticated approach, using the formal relationship between the $S$ matrix and field-theoretic $N$-point functions and the accurate perturbative expansion in the photon-quark coupling to express the photon as a superposition of QCD eigenstates.

The concept and realization is lucidly presented by $\mathrm{Ji}$ and Jung in Refs. [1,2], where they consider the hadronic structure of the photon using lattice three-point functions. We will outline the method as applied to meson twophoton decays.

The amplitude for the two-photon decay of a meson $M$ can be expressed in terms of a photon two-point function in Minkoswki space by means of the Lehmann-SymanzikZimmermann reduction

$$
\left\langle\gamma\left(q_{1}, \lambda_{1}\right) \gamma\left(q_{2}, \lambda_{2}\right) \mid M(p)\right\rangle=-\lim _{\substack{q_{1}^{\prime} \rightarrow q_{1} \\ q_{2}^{\prime} \rightarrow q_{2}}} \epsilon_{\mu}^{*}\left(q_{1}, \lambda_{1}\right) \epsilon_{\nu}^{*}\left(q_{2}, \lambda_{2}\right) q_{1}^{\prime 2} q_{2}^{\prime 2} \int d^{4} x d^{4} y e^{i q_{1}^{\prime} \cdot y+i q_{2}^{\prime} \cdot x}\left\langle 0\left|T\left\{A^{\mu}(y) A^{\nu}(x)\right\}\right| M(p)\right\rangle,
$$

up to photon renormalization factors. The explicit photon fields prevent direct computation of this quantity in lattice QCD; however, we can utilize the perturbative expansion of the photon-quark coupling to approximately integrate them out (the path integral over gluon fields is suppressed):

$$
\begin{aligned}
\int \mathcal{D} A \mathcal{D} \bar{\psi} \mathcal{D} \psi e^{i S_{\mathrm{QED}}[A, \bar{\psi}, \psi]} A^{\mu}(y) A^{\nu}(x)= & \int \mathcal{D} A \mathcal{D} \bar{\psi} \mathcal{D} \psi e^{i S_{0}[A, \bar{\psi}, \psi]}\left(\ldots+\frac{e^{2}}{2} \int d^{4} z d^{4} w\left[\bar{\psi}(z) \gamma^{\rho} \psi(z) A_{\rho}(z)\right]\right. \\
& \left.\times\left[\bar{\psi}(w) \gamma^{\sigma} \psi(w) A_{\sigma}(w)\right]+\ldots\right) A^{\mu}(y) A^{\nu}(x) .
\end{aligned}
$$

The integration over the photon field can be carried out by Wick contracting the fields into propagator products, so that, neglecting disconnected pieces,

$$
\begin{aligned}
\left\langle\gamma\left(q_{1}, \lambda_{1}\right) \gamma\left(q_{2}, \lambda_{2}\right) \mid M(p)\right\rangle= & \left(-e^{2}\right) \lim _{\substack{q_{\prime}^{\prime} \rightarrow q_{1} \\
q_{2}^{\prime} \rightarrow q_{2}}} \epsilon_{\mu}^{*}\left(q_{1}, \lambda_{1}\right) \epsilon_{\nu}^{*}\left(q_{2}, \lambda_{2}\right) q_{1}^{\prime 2} q_{2}^{\prime 2} \int d^{4} x d^{4} w d^{4} z e^{i q_{1}^{\prime} \cdot x} D^{\mu \rho}(0, z) D^{\nu \sigma}(x, w) \\
& \times\left\langle 0\left|T\left\{j_{\rho}(z) j_{\sigma}(w)\right\}\right| M(p)\right\rangle .
\end{aligned}
$$


The photon propagator can be written $D^{\mu \nu}(0, z)=-i g^{\mu \nu} \int\left[d^{4} k /(2 \pi)^{4}\right]\left[e^{i k . z} /\left(k^{2}+i \epsilon\right)\right]$, cancelling the inverse propagators outside the integral.

As explained in Ref. [1], the resulting expression can be analytically continued from Minkowski to Euclidean spacetime provided the photon virtualities $Q_{1}^{2}=\left|\vec{q}_{1}\right|^{2}-\omega_{1}^{2}$ and $Q_{2}^{2}=\left|\vec{q}_{2}\right|^{2}-\omega_{2}^{2}$ are not sufficiently timelike that they can produce on-shell hadrons. In charmonium [3], this limits us to $Q^{2}>-m_{J / \psi}^{2}$. Using a suitable QCD interpolating field to produce $M$ and reversing the operator time ordering for convenience, we have

$$
\begin{aligned}
\left\langle M(p) \mid \gamma\left(q_{1}, \lambda_{1}\right) \gamma\left(q_{2}, \lambda_{2}\right)\right\rangle= & \lim _{t_{f}-t \rightarrow \infty} e^{2} \frac{\epsilon_{\mu}\left(q_{1}, \lambda_{1}\right) \epsilon_{\nu}\left(q_{2}, \lambda_{2}\right)}{\frac{Z_{M}(p)}{2 E_{M}(p)} e^{-E_{M}(p)\left(t_{f}-t\right)}} \int d t_{i} e^{-\omega_{1}\left(t_{i}-t\right)}\langle 0| T\left\{\int d^{3} \vec{x} e^{-i \vec{p} \cdot \vec{x}} \varphi_{M}\left(\vec{x}, t_{f}\right)\right. \\
& \left.\times \int d^{3} \vec{y} e^{i \vec{q}_{2} \cdot \vec{y}} j^{\nu}(\vec{y}, t) j^{\mu}\left(\overrightarrow{0}, t_{i}\right)\right\}|0\rangle .
\end{aligned}
$$

It is clear from the previous discussion that obtaining two-photon widths is a natural extension to the study of radiative transitions carried out in Ref. [4]-there we computed three-point functions involving vector currents with the source $\left(t_{i}\right)$ and sink $\left(t_{f}\right)$ positions fixed and varied the vector current "insertion" $(t)$ across the temporal direction to plot out a plateau. For two-photon widths, we repeat this but with a varying sink [or, in the case of Eq. (1), source] position which will be integrated over with an exponential weighting.

Our calculation was performed in the quenched truncation of QCD using the CHROMA software system. We employed a $24^{3} \times 48$ lattice generated using a Wilson gauge action with coupling $\beta=6.5$ and lattice spacing $a \approx 0.047 \mathrm{fm}$ (determined from the static quark potential in Ref. [5]). Quark propagators were computed using a nonperturbatively improved Clover action [6] with Dirichlet boundaries in the temporal direction, with the charm quark mass set using the spin-averaged $\eta_{c}, J / \psi$ mass, accurate to within 3\%. Since we adopted only smeared local fermion bilinears as meson interpolating fields, we had access to three-point functions involving $\eta_{c}$ and $\chi_{c 0}$ mesons. In Ref. [4], typical radii of charmonium states were extracted and found to be much smaller than the $\sim 1.2 \mathrm{fm}$ box used here. We adopt the conserved ("point-split") vector current at the insertion-our experience in radiative transitions is that including the improvement term [7] has an effect below 5\% near $Q^{2}=0$.

We applied two different methods to calculate (1). The first (using 174 configurations) was to place the meson state at a fixed sink position $t_{f}=37$. As in Ref. [4], the sink was used as a sequential source for a backward propagator inversion, meaning that its properties were fixed for each computation while we were able, without further cost, to vary the direction and momentum of the insertion and the direction of the source field. We then computed with all possible source positions $t_{i}$, which, while costly in computing time, allowed us to freely vary the value of $\omega_{1}$ and, hence, $Q_{1}^{2}$ and, in addition, to view the subsequent integrand. In Fig. 1(a), we display the integrand for insertion positions $t=4,16,32, \vec{p}_{f}=(000)$, and $\vec{q}_{1}=$ (100) with an $\eta_{c}$ at the sink.

It is clear that, provided the insertion is not placed too close to the Dirichlet wall (i.e., $t \gtrsim 7$ ), we will be able to capture the full integral by summing time slices $t_{i}$. The integral as a function of insertion position $t$ is shown in Fig. 1(b) for a selection of $Q_{2}^{2}$ with $Q_{1}^{2}=0\left(\omega_{1}=\left|\vec{q}_{1}\right|\right)$ where we observe plateaus with the deviation from plateau behavior at larger $t$ coming from excited $\eta_{c}$ contributions, both of which are fitted simultaneously. Extracting the plateau values for a range of $Q_{1}^{2}$ (which we are free to choose continuously) and $Q_{2}^{2}$ (which is fixed for a given set of $\omega_{1}, \vec{q}_{2}, \vec{p}$ ), we find the dependence displayed in Fig. 2. We plot dimensionless $F$ defined by $\left\langle\eta_{c} \mid \gamma\left(q_{1}, \lambda_{1}\right) \gamma\left(q_{2}, \lambda_{2}\right)\right\rangle=2\left(\frac{2}{3} e\right)^{2} m_{\eta_{c}}^{-1} F\left(Q_{1}^{2}, Q_{2}^{2}\right) \times$ $\epsilon_{\mu \nu \rho \sigma} \epsilon_{1}^{\mu} \epsilon_{2}^{\nu} q_{1}^{\rho} q_{2}^{\sigma}$, where the on-shell decay width is $\Gamma\left(\eta_{c} \rightarrow \gamma \gamma\right)=\pi \alpha_{e m}^{2} \frac{16}{81} m_{\eta_{c}}|F(0,0)|^{2}$.
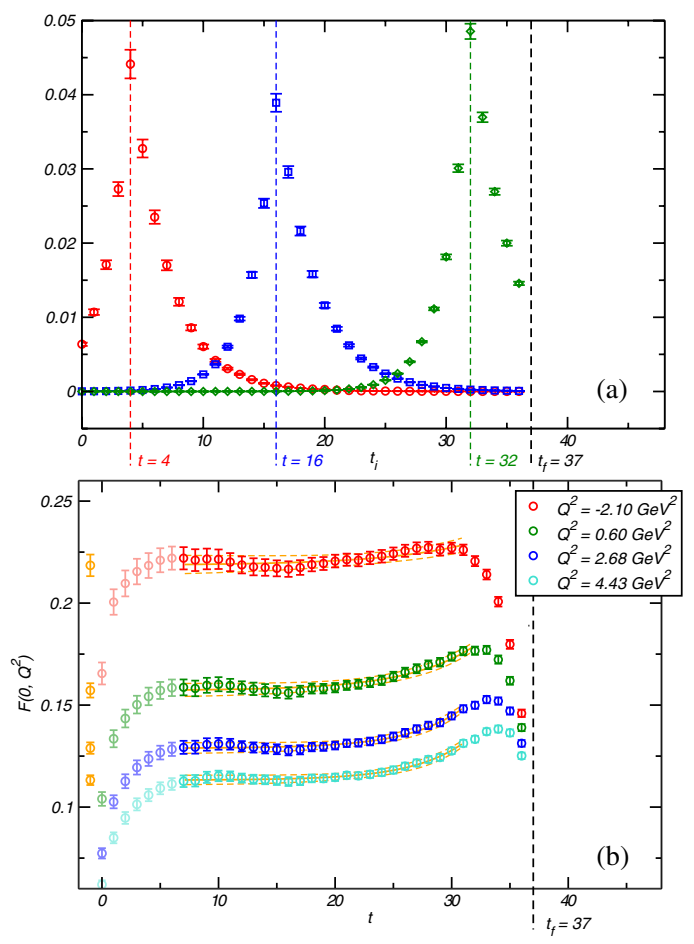

FIG. 1 (color online). (a) Integrand in (1) at three values of vector current insertion time $(t=4,16,32)$ with a pseudoscalar sequential source at sink position $t_{f}=37$. (b) Pseudoscalar twophoton form factor as a function of time slice $t$ from (1). The first six time slices are ghosted out due to the Dirichlet wall truncating the integral. Constant plus single exponential fits are shown as continuous curves. 
In order to more clearly express the voluminous data in Fig. 2, we adopt a simple one-pole parametrization to fit the data for each value of $Q_{1}^{2}$ :

$$
F\left(Q_{1}^{2}, Q_{2}^{2}\right)=\frac{\hat{F}\left(Q_{1}^{2}, 0\right)}{1+\frac{Q_{2}^{2}}{\mu^{2}\left(Q_{1}^{2}\right)}} .
$$

The curves in Fig. 2 are fits of this form. In the insets in Fig. 2, we display the fit parameters $\hat{F}\left(Q_{1}^{2}, 0\right), \mu\left(Q_{1}^{2}\right)$. $\hat{F}\left(Q_{1}^{2}, 0\right)$ is itself then fitted with a one-pole form with $\hat{F}(0,0)=0.168(4), \hat{\mu}=3.040(20) \mathrm{GeV}$, which is compatible with $\mu(0)=3.008(16) \mathrm{GeV}$, indicating the reasonableness of the one-pole parametrization in this $Q^{2}$ region. The both-photons on-shell point is 3 standard deviations smaller than the value extracted from the Particle Data Group (PDG) [8] decay width 0.274(43).

The error quoted on our result is statistical only and must be augmented by an uncertainty due to scaling from our fixed lattice spacing to the continuum and one related to the lack of light-quark loops within the quenched truncation. Since we compute at only one lattice spacing, we cannot accurately determine a scaling uncertainty; however, we expect the nonperturbatively tuned Clover action to have small $\mathcal{O}(a)$ scaling errors. On the other hand, it is possible that we have non-negligible $\mathcal{O}\left(m_{c} a\right)$ scaling which we conservatively estimate to be at the $15 \%$ level.

In the NRQCD factorized approach, the decay amplitude is proportional to the $\eta_{c}$ wave function at the origin with relativistic corrections. In the quenched theory utilized here, the incorrect running of the coupling down to short distances with the scale set at long distances does typically lead to a depletion of the wave function at the ori-
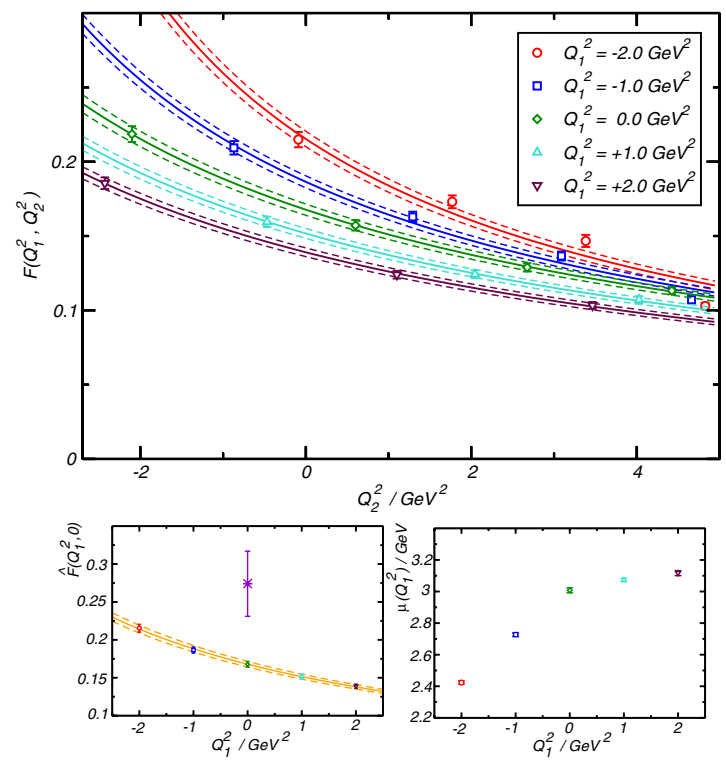

FIG. 2 (color online). $\quad \eta_{c}$ two-photon form factor $F\left(Q_{1}^{2}, Q_{2}^{2}\right)$. Points are lattice QCD data; fits are monopole forms as described in the text. The lower left shows fitted amplitudes at $Q_{2}^{2}=0$ and the lower right the fitted pole masses $\mu\left(Q_{1}^{2}\right)$. gin. On the lattice used here, we find [9] $f_{\psi}=386(6) \mathrm{MeV}$ in comparison to the experimental 411(7) MeV; however, we cannot determine to what degree the quenched depletion is offset by $\mathcal{O}\left(m_{c} a\right)$ scaling effects, and as such we assign an estimated $10 \%$ error due to quenching.

The similarity of $\mu(0)$ to the $J / \psi$ mass in our calculation [3006(5) MeV] suggests an approximate vector meson dominance description of the form factor. This is at first sight somewhat surprising, given that vector meson dominance (VMD) was demonstrated not to hold for the $\eta_{c}$ charge "form factor" in Ref. [4]. This was explained as being due to the presence of very many closely spaced vector poles (the $J / \psi, \psi^{\prime} \ldots$ ) which must be summed - in contrast to the light-quark sector where the $\rho(1450)$ is rather distant in comparison to the $\rho(770)$ and where VMD tends to be a good approximation.

What we suspect is happening here is that, while the excited $\psi$ poles are not negligible by their remoteness, they are almost negligible by their small residues. Consider the case $Q_{1}^{2}=0$ and the time ordering in which photon 1 is emitted from the $\eta_{c}$ first; then the time-dependent perturbation theory amplitude is proportional to

$$
\sum_{N} \frac{\left\langle\eta_{c}\left|j_{\mu}(0)\right| \psi^{(N)}\right\rangle\left\langle\psi^{(N)}\left|j_{\nu}(0)\right| 0\right\rangle}{m\left(\eta_{c}\right)-E\left(\psi^{(N)}\right)}
$$

The numerator is essentially the residue of the $N$ th excited $\psi$ pole and is seen to be proportional to the product of the $M 1$ transition matrix element between $\eta_{c}$ and $\psi^{(N)}$ and the decay constant of the $\psi^{(N)}$. The decay constants fall slowly with increasing $N$ as observed from the experimental $e^{+} e^{-}$ widths, while the $M 1$ transition amplitudes are expected to fall rather rapidly - they are "hindered" transitions that are proportional to the overlap of orthogonal wave functions with a small correction for recoil. Because of this, only the $J / \psi$ term in the sum has a considerable residue (since the wave function overlap with the $\eta_{c}$ is close to 1) and we observe something like VMD. A hint of this behavior was observed in the L3 experiment [10] and a model realization demonstrated in Ref. [11].

The fact that $\mu$ varies with $Q_{1}^{2}$ suggests that as this photon goes off-shell the higher $\psi$ resonances get a larger residue (through a larger recoil correction to the hindered transition) and contribute more to the sum over poles moving the effective pole position $\mu\left(Q_{1}^{2}\right)$.

The method of computing three-point functions with all possible source positions $\left(t_{i}=0 \ldots t_{f}\right)$ is extremely costly in computing resources. With the penalty of losing the ability to freely vary $Q_{1}^{2}$, we can reduce the computing time by a factor of $\mathcal{O}\left(L_{t}\right)$ by putting the meson interpolating field at the source and using

$$
\int d t e^{\omega_{1} t} \int d^{3} \vec{z} e^{i \vec{q}_{1} \cdot \vec{z}} \bar{\psi}(\vec{z}, t) \gamma^{\mu} \psi(\vec{z}, t)
$$

in the sequential source for the backward propagator inversion. It is then necessary to fix $\omega_{1}$ and $\vec{q}_{1}$ in advance, and one cannot view the integrand since the integration is 

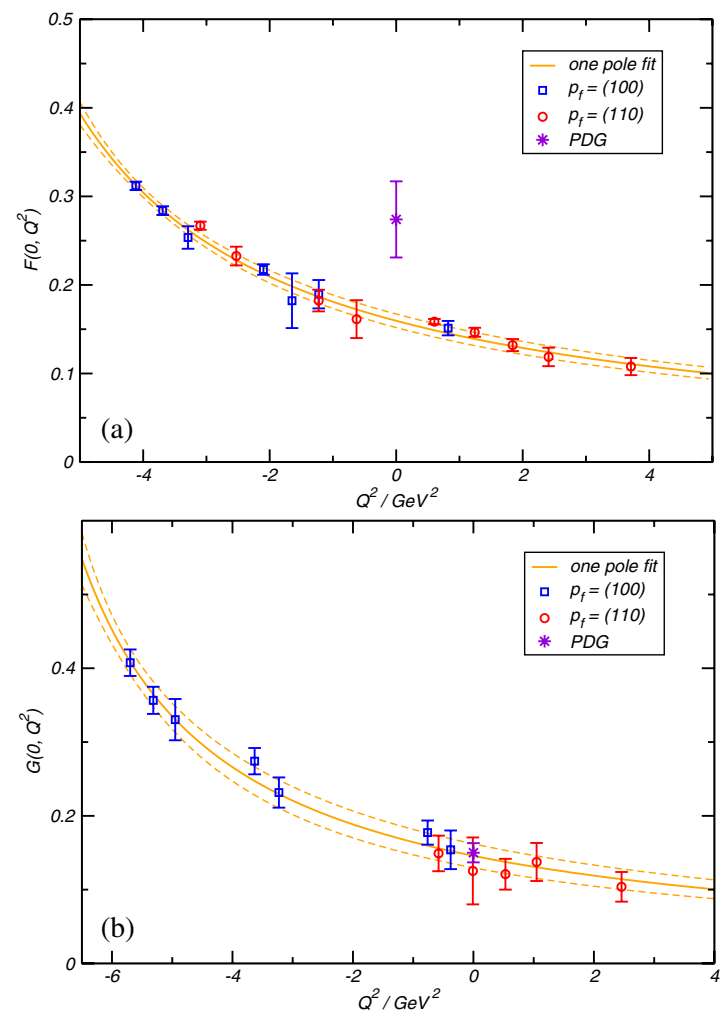

FIG. 3 (color online). (a) $\eta_{c} \rightarrow \gamma \gamma^{*}$ amplitude. (b) $\chi_{c 0} \rightarrow \gamma \gamma^{*}$ amplitude. Fits are monopole forms as described in the text.

being performed "on the fly" within the sequential source. We computed 300 configurations with $Q_{1}^{2}=0$ in this way, and the results for $\eta_{c}$ and $\chi_{c 0}$ are displayed in Fig. 3. Note that the one-pole fit to the $\eta_{c}$ data yields $F(0,0)=$ $0.160(8)$ and $\mu=2.899(71)$, values that are in agreement with those extracted from the method described above.

We define the $\chi_{c 0}$ two-photon form factor by

$$
\begin{aligned}
\left\langle\chi_{c 0} \mid \gamma\left(q_{1}, \lambda_{1}\right) \gamma\left(q_{2}, \lambda_{2}\right)\right\rangle= & 2\left(\frac{2}{3} e\right)^{2} m_{\chi_{c 0}}^{-1} G\left(Q_{1}^{2}, Q_{2}^{2}\right) \\
& \times\left(\epsilon_{1} \cdot \epsilon_{2} q_{1} \cdot q_{2}\right. \\
& \left.-\epsilon_{2} \cdot q_{1} \epsilon_{1} \cdot q_{1}\right) .
\end{aligned}
$$

The PDG [8] average of experimental measurements for the two-photon width corresponds to $|G(0,0)|=$ $0.150(13)$, with which we are in excellent agreementour single-pole fit to lattice data returns $|G(0,0)|=$ $0.146(16)$ and $\mu=2.976(81) \mathrm{GeV}$.

The logic used to justify the approximate $J / \psi$ VMD observed for the $\eta_{c}$ does not so obviously apply to the $\chi_{c 0}$. The radiative transitions to virtual vector meson states are now $E 1$ and are not hindered for the excited states to the same extent as $M 1$ (this can be seen in, for example, the quark model study [12]). Since the residues of excited state poles are not falling so quickly, they can contribute to the sum and we will not necessarily see dominance of the $J / \psi$.

Unfortunately, in this case the two nearest poles, the $\psi(3686)$ and the $\psi(3770)$, can conspire to cancel each other's effect. Using a combination of experimental data and quark model predictions [12], one estimates that the residues of these two poles are approximately equal (they have a value of about $1 / 3$ the $J / \psi$ residue). If these residues happen to have opposite sign, then the $J / \psi$ pole will dominate with small contributions from the more highly excited $\psi$ resonances. Our data are insufficiently precise to distinguish any deviation from a one-pole behavior.

In summary, we have demonstrated the feasibility of using a suitable sum over lattice time slices to simulate a photon in an external state, using the phenomenologically interesting case of the two-photon decays of charmonia. Our results $\Gamma\left(\eta_{c} \rightarrow \gamma \gamma\right)=2.65(26)_{\text {stat }}(80)_{\text {scal. }}$ (53) $)_{\text {cuen. }} \mathrm{keV}$ and $\Gamma\left(\chi_{c 0} \rightarrow \gamma \gamma\right)=2.41(58)_{\text {stat }}(72)_{\substack{\text { scal. } \\ \text { sys }}}(48)_{\substack{\text { suen. } \\ \text { sys }}}^{\text {sys }} \mathrm{keV}^{\text {sys }}$ are in reasonable agreement with experiment. We believe them to be systematics dominated, in particular, owing to the neglect of light-quark loops and discretization effects related to the heavy-quark mass. The systematic error can be reduced with further computations, which are now warranted given the feasibility demonstrated herein. This lattice technique has the advantage over nonrelativistic models that it can be applied to the light-quark sector without fundamental change.

Notice: Authored by The Southeastern Universities Research Association, Inc. under U.S. DOE Contract No. DE-AC05-84150. The U.S. Government retains a nonexclusive, paid-up, irrevocable, worldwide license to publish or reproduce this manuscript for U.S. Government purposes. Computations were performed on clusters at Jefferson Laboratory under the SciDAC initiative.

*Electronic address: dudek@jlab.org

[1] X.-d. Ji and C.-w. Jung, Phys. Rev. Lett. 86, 208 (2001).

[2] X.-d. Ji and C.-w. Jung, Phys. Rev. D 64, 034506 (2001).

[3] This is true within the quenched truncation, neglecting disconnected diagrams. Relaxing these approximations allows production of light vector mesons or vector glueballs-phenomenologically, we expect these states to have small coupling to the charmonium meson.

[4] J. J. Dudek, R. G. Edwards, and D. G. Richards, Phys. Rev. D 73, 074507 (2006).

[5] R. G. Edwards, U.M. Heller, and T. R. Klassen, Nucl. Phys. B517, 377 (1998).

[6] M. Luscher, S. Sint, R. Sommer, P. Weisz, and U. Wolff, Nucl. Phys. B491, 323 (1997).

[7] G. Martinelli, C. T. Sachrajda, and A. Vladikas, Nucl. Phys. B358, 212 (1991).

[8] S. Eidelman et al. (Particle Data Group), Phys. Lett. B 592, 1 (2004).

[9] The $\eta_{c}$ decay constant should be equal to the $J / \psi$ up to small spin-dependent corrections; see [4] for an explicit lattice extraction.

[10] M. Acciarri et al. (L3 Collaboration), Phys. Lett. B 461, 155 (1999).

[11] O. Lakhina and E. S. Swanson, Phys. Rev. D 74, 014012 (2006).

[12] T. Barnes, S. Godfrey, and E. S. Swanson, Phys. Rev. D 72, 054026 (2005). 